\title{
Implementation of extended high-gain observer in low-cost optitrack motion tracking system for UAV control
}

\begin{abstract}
The objective of this paper is to present the implementation procedure and experimental results of using Extended High-Gain Observer (EHGO) in estimating the states from low-cost Optitrack camera that is being used as the motion tracking in the Unmanned Aaerial Vehicle (UAV) system. Owing to the low-cost sensor, the signal produced is noisy which requires filters to produce a good signal. The standard procedure to obtain differentiated signal is complex. Therefore, this paper proposed an alternative method using EHGO. The theory of EHGO is presented, and the steps taken to transform EHGO algorithm from continuous to digital form are described. Validation is performed in open-loop and closed-loop flight test to analyze the performance. The results show that EHGO able to estimate the velocity state at the same performance quality as the standard procedure using Kalman filter. Nonetheless, the advantage of EHGO is due to its simple algorithm hence minimal parameters to tune.
\end{abstract}

Keyword: Extended high-gain observer (EHGO); Unmanned aaerial vehicle (UAV); Optitrack; Kalman filter 